Article

\title{
Examining the potential impacts of air pollution on China's in- bound tourism: The case of Beijing and Shanghai
}

\author{
Xu Ni ${ }^{1}$, Hongqing Ma $2, *$
}

1 School of Management and Economics, Kunming University of Science and Technology, Kunming, Yunan 650093, People's Republic of China; nixu618@126.com

2 Shandong Provincial Key Lab. of Soil Conservation and Environmental Protection, College of Resources and Environment, Linyi University, Linyi, Shandong 276000, People's Republic of China; hongqing6010@126.com

* Correspondence: hongqing6010@126.com; Tel. +8615753960209

\begin{abstract}
Concerns about China's air quality, and its impact on the important tourism industry have been on the debate in recent years. This article aims to investigate the potential effect of air pollution on direct economic impact of tourism, using the case of Beijing and Shanghai. The results indicate that air pollution negatively affects China's inbound tourism, resulting in huge loss of tourist arrivals and receipts, and Beijing suffers a greater loss in comparison with Shanghai, its loss in tourist number amounts to 1569,700 persons, equal to CNY 10264.268 million in tourism receipts, and the GDP losses ranges from CNY 20528.536 to 41057.072 across major source countries. This study provides a quantification of the impact helpful to generate a social awareness of air pollution detrimental impacts on inbound tourism and hence the economy.
\end{abstract}

Keywords: air pollution; inbound tourism; tourist arrivals; tourism receipts

\section{Introduction}

China's foreign tourist arrivals have declined slightly in the last few years for many reasons, one potential reason is the general perception of poor air quality in many major cities. As reported, some areas in China suffer from strong air pollution, especially in winter (Beckena., et.al, 2016) [1]. This situation undermines China's attractiveness as a tourist destination, and might have a detrimental effect on its inbound tourists inflow. Against this background, the precise objective of this research is to quantify the effect of air pollution on inbound tourists' inflows, and to provide an initial estimate of the associated direct economic impact.

In recent years, the impact of environmental deterioration and climate change on tourism industry has strongly erupted in the academic debate. A considerable amount of these studies indicated that environmental deterioration and climate change could impact tourism industry. Hamilton and Tol (2007) applied a simulation to indicate how climate change would reduce tourist arrivals in Germany, the UK and Ireland [2]. Álvarez-Díaz et al. (2010) studied the correlation between North Atlantic Oscillation (NAO) and inbound tourism to Balearic Islands [3]. They found a significant effect of changes in this atmosphere phenomenon on international arrivals. Sajjad et.al (2014) researched the longrun correlation among air pollution, climate change and tourism industry in the World's largest regions [4]. An increase in air pollution and climate change was found to have a negative impact on tourism activity. Zhang, et.al (2015) used questionnaires to study potential tourists' perception of air pollution's effects on tourism experience in Beijing [5]. They found that Haze pollution negatively affected travel perception. Becken, et.al (2016) surveyed Western tourists' contemporary views on air pollution in China [6]. The authors found that a deterioration of air quality adversely influnedced China's destination image and the intention to travel to the country. Xu and Reed (2017) applied a VAR model to prove a negative bidirectional relationship between perceived air pollution and Chinese 
inbound tourism [7]. Chen et al. (2017) investigated whether air pollution would impact tourism demand to Sun Moon Lake National Scenic Area at Taiwan [8]. They provided adequate evidence that air pollution would drive away tourists.

These articles, among others, have proven negatively impact of environmental deterioration and climate change on tourism industry. However, to the best of our knowledge, there is still a scarcity of papers providing an empirical quantification of the loss in tourism receipts caused by air pollution. Hence, this article tries to contribute to this gap, and represents three main contributions: (1) this is the first research to estimate the detrimental effect of air pollution on the direct economic contribution of tourism through its negative impact on arrivals. (2) this research documents new empirical evidence of the reasons for the reduction of inbound tourism. (3) the theoretical and empirical evaluation of noneconomic factors (environment factor) impact on inbound tourism demand contributes to the literature.

The rest of the article is organized as follows. Section 2 describes the theoretical model and data used in the paper. Section 3 presents the empirical results of the effect of air pollution on inbound tourism demand and its direct economic impact. Finally, concluding remarks and recommendations are presented at the last section.

\section{Theoretical model and data description}

\subsection{Theoretical model}

Consistent with the general literature mentioned in section 1, tourist arrivals are usually used to measure tourism demand. The most influential determinants of inbound tourism demand are tourist's income, relative real destination's price (at the destination relative to those in the source country, and relative real price at alternative destinations (Song \& Li, 2008) [9]. Thus, the standard tourism demand function is specified as:

$$
T A_{i t}=f\left(Y_{i t}, P_{i t}, P_{s t}, e_{i t}\right)
$$

where

$\mathrm{TA}_{\text {it }}$ denotes tourist arrivals to Beijing or Shanghai from source market $\mathrm{i}$ at time $\mathrm{t}$;

$Y_{i t}$ denotes tourist income at source market $i$ at time $t$;

$\mathrm{P}_{\mathrm{it}}$ is the real own price of tourism in mainland China relative to that in source market $\mathrm{i}$ at time $\mathrm{t}$;

$\mathrm{P}_{\text {it }}=\frac{C P I_{m c} / E X_{m c}}{C P I_{i} / E X_{i}}, C P I_{m c}$ and $C P I_{i}$ are the China's mainland consumer price indexes (CPI) and source market's consumer price indexes, respectively, $E X_{m c}$ and $E X_{i}$ are the corresponding nominal exchange rates.

$\mathrm{P}_{\text {st }}$ captures the price of substitute or competitive destination, which is a weighted index of selected areas or countries. According to the similarity with the geography and culture of mainland China, this paper chooses Thailand, South Korea, Singapore and Chinese Taiwan as the competition or substitute destinations of mainland China. Hence, $\mathrm{P}_{\mathrm{st}}=\sum_{j=1}^{4} \frac{C P I_{j}}{E X_{j}} w_{i j}, \mathrm{j}=1,2,3,4 ; w_{i j}$ is the proportion of inbound tourism arrivals from these destinations.

$e_{i t}$ is the error disturbance.

In order to capture the growth impacts (elasticity coefficients), Eq. (1) is expressed as a nature logarithm. Thus the long-term static function is defined:

$$
\ln T A_{i t}=\beta_{0}+\beta_{1} \ln Y_{i t}+\beta_{2} \ln P_{i t}+\beta_{3} \ln P_{s t}+u_{i t}
$$

Eq. (2) is a static long-run model, which just capture the long-run correlation between explanatory and dependent variables. But, in fact, when tourists make decisions to travel, they not only consider their current economic situation (tourist income, the price of destination, etc.), but also consider historical information (e.g. the word-of-mouth effect) and 
time lags (i.e. making plans in advance) (Song et al., 2012) [10]. Therefore, to capture the long-run and short-run relationships among variable, the unrestricted error correction model (UECM) is applied, as shown in Eq. (3):

$$
\begin{gathered}
\Delta \ln T A_{i t}=\alpha_{0}+\sum_{j=1}^{p} \alpha_{j} \Delta \ln T A_{i t-j}+\sum_{j=0}^{p} \beta_{j} \Delta \ln Y_{i t-j}+\sum_{j=0}^{p} \gamma_{j} \Delta \ln P_{i t-j}+\sum_{j=0}^{p} \delta_{j} \Delta \ln P_{s t-j}+\lambda_{1} \ln T A_{i t-}+\lambda_{2} \ln Y_{i t-1}+ \\
\lambda_{3} \ln P_{i t-1}+\lambda_{4} \ln P_{s t-1}+\varepsilon_{i t}
\end{gathered}
$$

where $p$ is the number of lags, which is decided by Akaike information criteria (AIC) and the Schwarz information criteria (SIC); $\varepsilon_{i t}$ is the error term assumed to follow a normal distribution.

In order to estimate the impact of air pollution on inbound tourism demand, this research identifies the events of high pollution by specifying a dummy variable that takes value 1 when the Air Quality Index (AQI) is greater than or equal to 101, and value 0 in "good air quality" periods ${ }^{1}$. When AQI is between 0 and 100, it indicates that the air quality is excellent and good, but more than or equal to 101 indicates air pollution.

Additional variables are incorporated leading to Eq. 4 to capture the effects of financial crisis and the deterministic seasonal pattern:

$$
\begin{gathered}
\Delta \operatorname{lnT} A_{i t}=\alpha_{0}+\sum_{j=1}^{p} \alpha_{j} \Delta \ln T A_{i t-j}+\sum_{j=0}^{p} \beta_{j} \Delta \ln Y_{i t-j}+\sum_{j=0}^{p} \gamma_{j} \Delta \ln P_{i t-j}+\sum_{j=0}^{p} \delta_{j} \Delta \ln P_{s t-}+\lambda_{1} \ln T A_{i t-1}+\lambda_{2} \ln Y_{i t-1}+ \\
\lambda_{3} \ln P_{i t-1}+\lambda_{4} \ln P_{s t-1}+D A Q I+D 08+\sum_{n=1}^{11} S_{i n} D_{n}+\varepsilon_{i t}
\end{gathered}
$$

Where

DAQI is the dummy for air quality (=1 AQI is greater than or equal to 101$)$, on which the coefficient controls for the effect of air pollution.

$\sum_{n=1}^{11} S_{i n}$ are the monthly seasonal dummies, which captures inbound tourism demand's seasonality.

D08 is the financial crisis dummy, which considers the effect of financial crisis.

Some advantages of this model are as follow. First, it can be used to a large or small size sample. Second, this model can examine both short-run and long-run correlation among tourism demand, air pollution and macroeconomic variables. A test-down procedure known as the general-to-specific method is followed to estimate the above equation, and then delete statistically insignificant variables and incorrectly signed. We will continue this procedure until achieve a satisfactory specific model. Although the general model includes several lagged variables and dummy variables, which reduce the Dof (degree of freedom), the final model does not have to be so redundant.

\subsection{Data description}

Shanghai and Beijing are examined, because they are the most popular inbound tourist cities in mainland China ${ }^{2}$ and are suffering from air pollution ${ }^{3}$. Six major source countries are examined given their high market shares in Shanghai and Beijing's inbound tourism market. The monthly data of tourist arrivals are obtained from the tourism bureau of statistics. The fiscal revenue from Wind database is used as a proxy for income variable $Y_{i t}$. The CPI indexes (Y2000=100) and the index of exchange rate (Y2000=100) are gained

1 AQI is between 0 and 100, which indicates that the air quality is excellent and good, but more than or equal to 101 indicate air pollution. Hence, for periods of AQI is greater than or equal to 101, the value was given as 1, while for other periods it was 0 .

2 Among all cities, Shanghai and Beijing are the first and second place in China's inbound market 2018, respectively.

3 Shanghai Municipal Environmental Protection Bureau reported that the excellent rate of AQI was 70.7\% in Shanghai, while it just 51 \% in Beijing according Beijing Municipal Environmental Protection Bureau report. 
from Wind database. All data are from January 2004 to December 2018. The AQI is from data center of the Ministry of Environmental Protection of China.

\section{The effects of air pollution}

In order to ensure all series are not integrated of order 2 or beyond, this paper carry out the Phillipse-Perron (PP) test and the Augmented Dickey Fuller (ADF) test. This research follows general-to-specific procedures to estimate the models. Table 1 and Table 2 report the final regression outputs. Table 1 exhibits that DAQI coefficients are negative and statistically significant for all series in Beijing at the $10 \%$ level, except South Korea, which indicate that air pollution of Beijing negative affect on inbound tourism demand from the six source markets, except South Korea market. DAQI coefficients of Shanghai are negative for all series, but only statistically significant for Asian source market (South Korea, Japan and Thailand) at the $10 \%$ level. The result implies that Shanghai's air pollution only negative impact on inbound tourism arrival in Asian source market (South Korea, Japan and Thailand), while the tourism arrivals from the US, the UK and Canada are not as affected as air pollution of Shanghai. These results suggest that the air pollution level influence the decision of travelers whether travel China or not. The negative effect implies that air pollution can reduce inbound tourism arrivals.

Table 1. Estimates of the demand models of Beijing's inbound tourism market

\begin{tabular}{|c|c|c|c|c|c|c|}
\hline & Canada & $\begin{array}{l}\text { South } \\
\text { Korea }\end{array}$ & Japan & Thailand & USA & UK \\
\hline $\mathrm{C}$ & $\begin{array}{l}-1.508 \\
(-1.439)\end{array}$ & $\begin{array}{l}2.343^{* * *} \\
(5.096)\end{array}$ & $\begin{array}{c}0.265 \\
(0.379)\end{array}$ & $\begin{array}{c}1.069^{* * *} \\
(3.333)\end{array}$ & $\begin{array}{c}5.540^{* * *} \\
(4.001)\end{array}$ & $\begin{array}{c}1.224 \\
(1.507)\end{array}$ \\
\hline LnTAit(-1) & $\begin{array}{c}-0.276^{* * *} \\
(-3.040)\end{array}$ & $\begin{array}{l}-0.155^{* *} \\
(-2.280)\end{array}$ & $\begin{array}{l}-0.077^{*} \\
(-1.733)\end{array}$ & $\begin{array}{l}-0.083^{*} \\
(-1.939)\end{array}$ & $\begin{array}{c}-0.584^{* * *} \\
(-4.606)\end{array}$ & $\begin{array}{c}-0.411^{* * *} \\
(-4.977)\end{array}$ \\
\hline $\operatorname{LnP} P_{i t}(-1)$ & $\begin{array}{l}-0.026 \\
(-0.077)\end{array}$ & $\begin{array}{l}-0.084^{*} \\
(-1.855)\end{array}$ & $\begin{array}{l}-0.158^{*} \\
(-1.837)\end{array}$ & $\begin{array}{c}0.314 \\
(1.092)\end{array}$ & $\begin{array}{c}-0.589^{* * *} \\
(-4.319)\end{array}$ & $\begin{array}{c}-0.205^{* * *} \\
(-3.543)\end{array}$ \\
\hline LnY $Y_{i t(-1)}$ & $\begin{array}{l}0.378^{* *} \\
(2.148)\end{array}$ & $\begin{array}{c}-0.088^{* * *} \\
(-2.791)\end{array}$ & $\begin{array}{c}0.034 \\
(0.827)\end{array}$ & $\begin{array}{l}0.054^{* *} \\
(6.156)\end{array}$ & $\begin{array}{c}0.025^{* * *} \\
(0.391)\end{array}$ & $\begin{array}{c}0.208^{* * *} \\
(3.881)\end{array}$ \\
\hline $\operatorname{LnPst}(-1)$ & $\begin{array}{l}-0.043 \\
(-0.092)\end{array}$ & $\begin{array}{c}-0.054 \\
(-0.244)\end{array}$ & $\begin{array}{l}-0.294 \\
(-1.277)\end{array}$ & $\begin{array}{l}-0.547^{*} \\
(1.789)\end{array}$ & $\begin{array}{l}-0.517^{* * *} \\
(-2.080)\end{array}$ & $\begin{array}{l}-0.509^{* * *} \\
(-3.450)\end{array}$ \\
\hline D(LnTA(- & $\begin{array}{c}-0.577^{* * *} \\
(-4.334)\end{array}$ & $\begin{array}{l}-0.765^{* * *} \\
(-15.925)\end{array}$ & $\begin{array}{c}0.372^{* * *} \\
(2.697)\end{array}$ & $\begin{array}{l}-0.902^{* * *} \\
(-25.765)\end{array}$ & $\begin{array}{c}-0.286^{* * *} \\
(-2.680)\end{array}$ & $\begin{array}{l}0.186^{*} \\
(1.684)\end{array}$ \\
\hline 1)) & $\begin{array}{l}-0.376^{* * *} \\
(-3.249)\end{array}$ & & & & & $\begin{array}{c}-0.275^{* * *} \\
(-2.671)\end{array}$ \\
\hline $\begin{array}{c}\mathrm{D}(\operatorname{LnTA}(- \\
2))\end{array}$ & $\begin{array}{c}-0.283^{* * *} \\
(2.972)\end{array}$ & & & & & \\
\hline
\end{tabular}




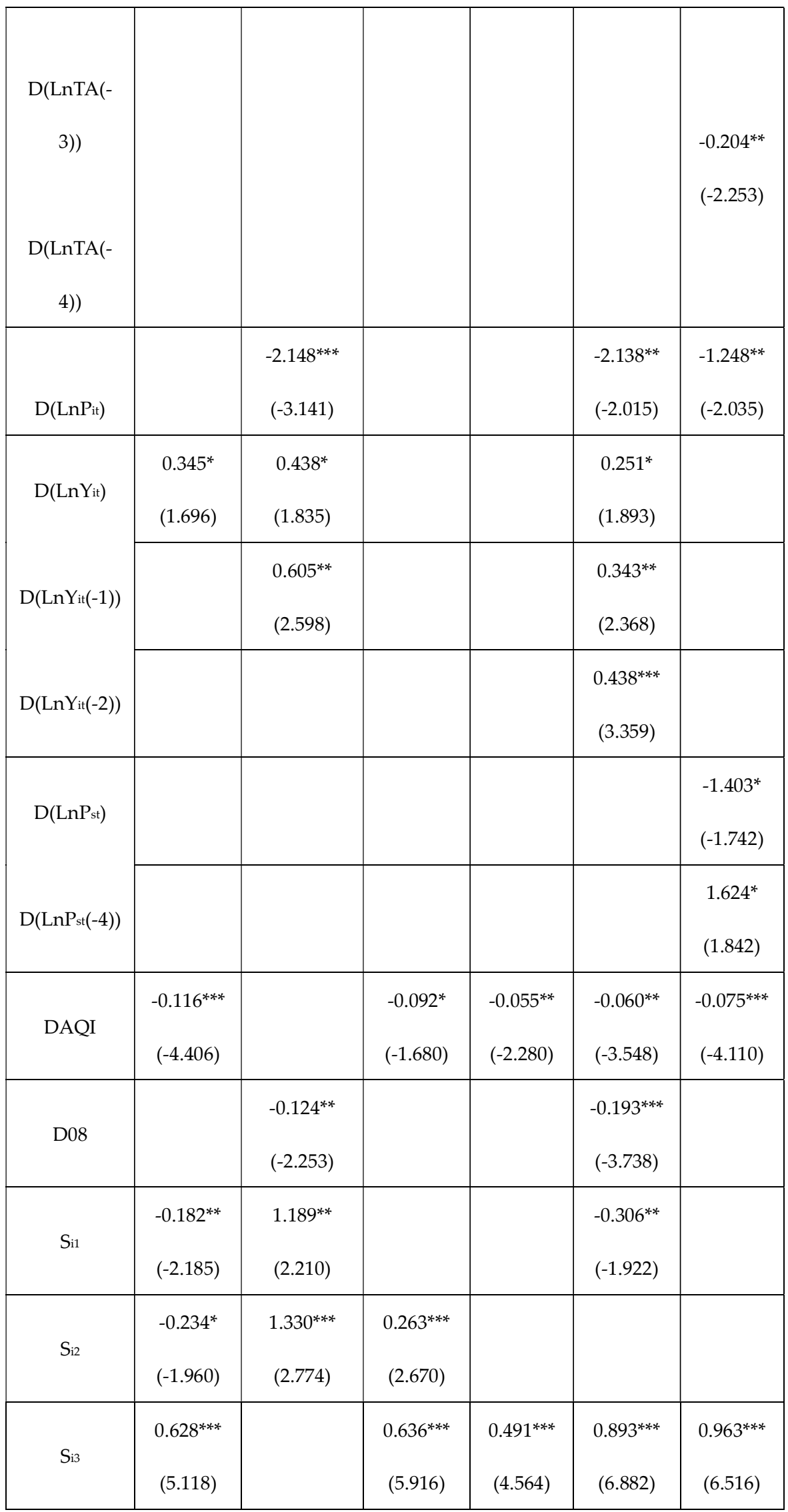




\begin{tabular}{|c|c|c|c|c|c|c|}
\hline $\mathrm{Si}_{\mathrm{i}}$ & $\begin{array}{l}0.793^{* * *} \\
(6.350)\end{array}$ & & & $\begin{array}{c}0.748^{* * *} \\
(7.094)\end{array}$ & $\begin{array}{c}0.723^{* * *} \\
(5.661)\end{array}$ & \\
\hline $\mathrm{Si}_{\mathrm{i} 5}$ & $\begin{array}{c}0.795^{* * *} \\
(7.858)\end{array}$ & & $\begin{array}{c}0.424^{* * *} \\
(3.372)\end{array}$ & & $\begin{array}{l}0.318^{*} \\
(1.736)\end{array}$ & $\begin{array}{c}0.894^{* * *} \\
(3.759)\end{array}$ \\
\hline Si6 & $\begin{array}{c}0.448^{* * *} \\
(4.711)\end{array}$ & & $\begin{array}{l}0.175^{*} \\
(1.957)\end{array}$ & $\begin{array}{c}-0.519^{* * *} \\
(-4.785)\end{array}$ & $\begin{array}{l}0.315^{* *} \\
(2.411)\end{array}$ & $\begin{array}{l}0.193^{*} \\
(1.740)\end{array}$ \\
\hline $\mathrm{Si} 7$ & $\begin{array}{c}0.353^{* * *} \\
(5.361)\end{array}$ & & $\begin{array}{l}0.179^{*} \\
(1.962)\end{array}$ & $\begin{array}{c}-0.408^{* * *} \\
(-2.974)\end{array}$ & $\begin{array}{l}0.604^{* * *} \\
(5.072)\end{array}$ & $\begin{array}{l}0.389^{* *} \\
(2.438)\end{array}$ \\
\hline $\mathrm{Si} 8$ & $\begin{array}{l}0.342^{* * *} \\
(4.064)\end{array}$ & $\begin{array}{l}0.311^{* * *} \\
(3.430)\end{array}$ & $\begin{array}{l}0.415^{* * *} \\
(4.980)\end{array}$ & & $\begin{array}{l}0.206^{*} \\
(1.844)\end{array}$ & $\begin{array}{l}0.547^{* *} \\
(2.613)\end{array}$ \\
\hline Si 9 & $\begin{array}{l}0.522^{* * *} \\
(6.126)\end{array}$ & & & $\begin{array}{l}0.257^{* *} \\
(2.241)\end{array}$ & $\begin{array}{l}0.452^{* * *} \\
(8.029)\end{array}$ & $\begin{array}{c}0.774^{* * *} \\
(6.557)\end{array}$ \\
\hline Si10 & $\begin{array}{l}0.834^{* * *} \\
(10.424)\end{array}$ & $\begin{array}{l}0.170^{*} \\
(1.839)\end{array}$ & $\begin{array}{l}0.158^{* *} \\
(2.023)\end{array}$ & $\begin{array}{c}0.459^{* * *} \\
(3.311)\end{array}$ & $\begin{array}{l}0.555^{* * *} \\
(4.436)\end{array}$ & $\begin{array}{l}0.357^{*} \\
(1.727)\end{array}$ \\
\hline Si11 & $\begin{array}{l}0.569^{* * *} \\
(9.170)\end{array}$ & $\begin{array}{l}0.271^{* * *} \\
(4.082)\end{array}$ & $\begin{array}{l}0.314^{* * *} \\
(2.672)\end{array}$ & $\begin{array}{l}0.192^{*} \\
(1.872)\end{array}$ & $\begin{array}{l}0.337^{* * *} \\
(2.766)\end{array}$ & $\begin{array}{l}0.499^{* *} \\
(2.158)\end{array}$ \\
\hline$R^{2}$ & 0.911 & 0.680 & 0.676 & 0.776 & 0.924 & 0.942 \\
\hline $\begin{array}{c}\text { Adjusted } \\
R^{2}\end{array}$ & 0.881 & 0607 & 0.591 & 0.728 & 0.904 & 0.919 \\
\hline AIC & -1.375 & -1.062 & -0.832 & -0.469 & -1.593 & -1.614 \\
\hline SIC & -0.636 & -0.498 & -0.202 & 0.073 & -0.942 & -0.783 \\
\hline F-statistic & 31.118 & 9.309 & 7.928 & 16.121 & 44.390 & 41.871 \\
\hline D-W & 2.008 & 1.974 & 2.009 & 1.727 & 1.974 & 1.945 \\
\hline$L M_{s c}$ & 2.427 & 0.000 & 0.423 & 2.464 & 0.791 & 7.710 \\
\hline $\mathrm{ARCH}$ & 3.341 & 0.319 & 1.308 & 0.240 & 0.596 & 5.799 \\
\hline JB & $13.948^{* * *}$ & 0.335 & $40.610^{* * *}$ & 0.952 & $68.880^{* * *}$ & $8.771^{* *}$ \\
\hline
\end{tabular}


Table 2. Estimates of the demand models of Shanghai's inbound tourism market

\begin{tabular}{|c|c|c|c|c|c|c|}
\hline & Canada & $\begin{array}{l}\text { South } \\
\text { Korea }\end{array}$ & Japan & Thailand & USA & UK \\
\hline $\mathrm{C}$ & $\begin{array}{l}-2.231^{*} \\
(-1.699)\end{array}$ & $\begin{array}{c}0.901 \\
(0.785)\end{array}$ & $\begin{array}{c}1.158 \\
(0.677)\end{array}$ & $\begin{array}{l}0.366 \\
(0.904)\end{array}$ & $\begin{array}{l}2.455^{* *} \\
(2.153)\end{array}$ & $\begin{array}{c}0.444 \\
(0.164)\end{array}$ \\
\hline $\operatorname{LnTA}_{\text {it }}(-1)$ & $\begin{array}{c}-0.248^{* * *} \\
(-2.960)\end{array}$ & $\begin{array}{c}-0.880^{* * *} \\
(-7.647)\end{array}$ & $\begin{array}{l}-0.209 \\
(-1.445)\end{array}$ & $\begin{array}{l}0.973^{* * *} \\
(25.944)\end{array}$ & $\begin{array}{l}-0.318^{* * *} \\
(-3.409)\end{array}$ & $\begin{array}{l}-1.296^{* * *} \\
(-5.583)\end{array}$ \\
\hline $\operatorname{LnP} P_{i t}(-1)$ & $\begin{array}{c}0.486 \\
(1.185)\end{array}$ & $\begin{array}{l}-0.260 \\
(-1.526)\end{array}$ & $\begin{array}{l}-0.050 \\
(-0.482)\end{array}$ & $\begin{array}{l}0.656 \\
(1.556)\end{array}$ & $\begin{array}{l}-0.280^{* * *} \\
(-2.667)\end{array}$ & $\begin{array}{l}-0.864^{* * *} \\
(-3.610)\end{array}$ \\
\hline $\operatorname{LnY}_{i t}(-1)$ & $\begin{array}{l}0.392^{*} \\
(1.972)\end{array}$ & $\begin{array}{l}0.690^{* * *} \\
(5.074)\end{array}$ & $\begin{array}{l}0.097^{* *} \\
(2.150)\end{array}$ & $\begin{array}{l}-0.052 \\
(-0.115)\end{array}$ & $\begin{array}{c}0.036 \\
(0.488)\end{array}$ & $\begin{array}{l}1.027^{* * *} \\
(3.608)\end{array}$ \\
\hline $\operatorname{LnP}_{\text {st }}(-1)$ & $\begin{array}{l}1.049^{*} \\
(1.864)\end{array}$ & $\begin{array}{l}-0.008 \\
(-0.017)\end{array}$ & $\begin{array}{l}-0.514^{*} \\
(-1.686)\end{array}$ & $\begin{array}{l}1.049^{* *} \\
(2.374)\end{array}$ & $\begin{array}{l}-0.172 \\
(-0.807)\end{array}$ & $\begin{array}{l}-0.219 \\
(-0.417)\end{array}$ \\
\hline $\begin{array}{c}\mathrm{D}(\operatorname{LnTA}(-1)) \\
\quad=-8643\end{array}$ & $\begin{array}{l}-0.503^{* * *} \\
(-4.786)\end{array}$ & $\begin{array}{l}0.319^{* * *} \\
(2.972)\end{array}$ & $\begin{array}{c}0.206 \\
(1.622)\end{array}$ & $\begin{array}{l}-0.552^{* * *} \\
(-5.604)\end{array}$ & $\begin{array}{l}-0.332^{* * *} \\
(-3.858)\end{array}$ & $\begin{array}{l}0.473^{* *} \\
(2.340)\end{array}$ \\
\hline $\mathrm{D}(\operatorname{LnTA}(-2))$ & & $\begin{array}{l}0.378^{* * *} \\
(4.703)\end{array}$ & $\begin{array}{l}-0.237^{* *} \\
(-2.129)\end{array}$ & & & \\
\hline $\mathrm{D}(\operatorname{LnTA}(-3))$ & & & $\begin{array}{l}0.177^{*} \\
(-1.738)\end{array}$ & & & \\
\hline $\begin{array}{l}\mathrm{D}(\operatorname{LnTA}(- \\
4))\end{array}$ & & & $\begin{array}{c}-0.301^{* * *} \\
(-3.026)\end{array}$ & & & \\
\hline $\mathrm{D}\left(\operatorname{Ln} P_{\mathrm{it}}\right)$ & & $\begin{array}{l}-0.858^{*} \\
(-1.962)\end{array}$ & & & $\begin{array}{l}-1.748^{* *} \\
(-2.124)\end{array}$ & \\
\hline $\mathrm{D}\left(\operatorname{Ln} P_{\mathrm{it}}(-1)\right)$ & & $\begin{array}{c}1.352^{* * *} \\
(2.873)\end{array}$ & $\begin{array}{l}1.068^{* *} \\
(1.997)\end{array}$ & & & $\begin{array}{c}1.556^{* * *} \\
(3.180)\end{array}$ \\
\hline $\mathrm{D}\left(\operatorname{Ln} P_{\mathrm{it}}(-2)\right)$ & & $\begin{array}{l}1.243^{* *} \\
(2.596)\end{array}$ & & & & \\
\hline $\mathrm{D}\left(\operatorname{Ln} \mathrm{Pit}_{\mathrm{it}}(-3)\right)$ & & & & & & $0.944^{* *}$ \\
\hline
\end{tabular}




\begin{tabular}{|c|c|c|c|c|c|c|}
\hline & & & & & & (2.017) \\
\hline $\mathrm{D}(\operatorname{Ln} Y i t)$ & & & & & $\begin{array}{l}0.233^{* *} \\
(2.427)\end{array}$ & \\
\hline $\mathrm{D}\left(\operatorname{LnY} Y_{\mathrm{it}(-1))}\right.$ & & & $\begin{array}{l}-0.083^{* *} \\
(-2.000)\end{array}$ & & & $\begin{array}{l}-0.942^{* * *} \\
(-3.233)\end{array}$ \\
\hline $\mathrm{D}\left(\operatorname{Ln} Y_{\mathrm{it}(-2))}\right.$ & & $\begin{array}{l}-0.943^{* * *} \\
(-6.476)\end{array}$ & $\begin{array}{l}-0.096^{* *} \\
(-2.300)\end{array}$ & & & $\begin{array}{l}-0.638^{* *} \\
(-2.532)\end{array}$ \\
\hline $\mathrm{D}\left(\operatorname{LnY} Y_{\mathrm{it}(-3))}\right.$ & & & $\begin{array}{l}-0.062^{*} \\
(-1.862)\end{array}$ & & & \\
\hline $\mathrm{D}(\operatorname{LnPst}(-2))$ & & & & $\begin{array}{l}-3.646^{* *} \\
(-1.986)\end{array}$ & & \\
\hline D(LnPst(-3)) & & & & & & $\begin{array}{l}-1.597^{* *} \\
(-2.179)\end{array}$ \\
\hline D(LnPst(-4)) & & & $\begin{array}{l}1.662^{* *} \\
(2.066)\end{array}$ & & & \\
\hline DAQI & & $\begin{array}{l}-0.076^{* * *} \\
(-2.640)\end{array}$ & $\begin{array}{l}-0.045^{* *} \\
(-1.995)\end{array}$ & $\begin{array}{l}-0.107^{* *} \\
(-2.416)\end{array}$ & & \\
\hline Si1 & & & & & $\begin{array}{l}0.163^{* *} \\
(2.144)\end{array}$ & \\
\hline $\mathrm{Si}_{\mathrm{i} 2}$ & & $\begin{array}{l}0.879^{* *} \\
(2.326)\end{array}$ & & $\begin{array}{l}0.194^{*} \\
(1.766)\end{array}$ & $\begin{array}{l}0.319^{* *} \\
(2.425)\end{array}$ & \\
\hline $\mathrm{Si} 3$ & $\begin{array}{l}0.827^{* * *} \\
(6.736)\end{array}$ & $\begin{array}{l}-0.636^{*} \\
(-1.668)\end{array}$ & $\begin{array}{l}0.481^{* * *} \\
(3.740)\end{array}$ & $\begin{array}{l}0.647^{* * *} \\
(6.116)\end{array}$ & $\begin{array}{l}0.741^{* * *} \\
(7.868)\end{array}$ & $\begin{array}{l}0.249^{*} \\
(1.907)\end{array}$ \\
\hline $\mathrm{Si}_{\mathrm{i}}$ & $\begin{array}{l}0.893^{* * *} \\
(6.478)\end{array}$ & $\begin{array}{l}0.500^{* * *} \\
(7.337)\end{array}$ & & $\begin{array}{l}0.792^{* * *} \\
(6.767)\end{array}$ & $\begin{array}{l}0.537^{* * *} \\
(6.379)\end{array}$ & \\
\hline $\mathrm{Si} 5$ & $\begin{array}{l}0.542^{* * *} \\
(5.197)\end{array}$ & $\begin{array}{l}0.120^{* * *} \\
(6.651)\end{array}$ & & & $\begin{array}{l}0.559^{* * *} \\
(5.004)\end{array}$ & $\begin{array}{l}0.250^{*} \\
(1.930)\end{array}$ \\
\hline Sir & $0.332^{* * *}$ & $0.796^{* * *}$ & $0.195^{* *}$ & & $0.492^{* * *}$ & $0.234^{* * *}$ \\
\hline
\end{tabular}




\begin{tabular}{|c|c|c|c|c|c|c|}
\hline & (3.743) & (5.963) & $(2.038)$ & & (6.356) & $(3.430)$ \\
\hline $\mathrm{S}_{\mathrm{i} 7}$ & $\begin{array}{l}0.417^{* * *} \\
(4.765)\end{array}$ & $\begin{array}{c}0.584^{* * *} \\
(5.645)\end{array}$ & $\begin{array}{l}0.271^{* *} \\
(2.414)\end{array}$ & & $\begin{array}{c}0.456^{* * *} \\
(5.044)\end{array}$ & \\
\hline Si8 & $\begin{array}{c}0.379^{* * *} \\
(3.458)\end{array}$ & $\begin{array}{c}0.492^{* * *} \\
(5.637)\end{array}$ & $\begin{array}{l}0.130^{*} \\
(1.678)\end{array}$ & $\begin{array}{c}0.251^{*} \\
(1.784)\end{array}$ & $\begin{array}{c}0.359^{* * *} \\
(4.477)\end{array}$ & $\begin{array}{c}0.322^{* * *} \\
(2.844)\end{array}$ \\
\hline Sig & $\begin{array}{c}0.712^{* * *} \\
(6.801)\end{array}$ & $\begin{array}{c}0.245^{* * *} \\
(3.999)\end{array}$ & $\begin{array}{c}0.347^{* * *} \\
(2.781)\end{array}$ & $\begin{array}{c}0.531^{* * *} \\
(4.504)\end{array}$ & $\begin{array}{c}0.3884^{* * *} \\
(9.550)\end{array}$ & $\begin{array}{c}0.443^{* * *} \\
(5.565)\end{array}$ \\
\hline$S_{i 10}$ & $\begin{array}{c}0.958^{* * *} \\
(9.641)\end{array}$ & $\begin{array}{c}0.240^{* * *} \\
(3.425)\end{array}$ & & $\begin{array}{c}0.554^{* * *} \\
(4.432)\end{array}$ & $\begin{array}{c}0.721^{* * *} \\
(7.654)\end{array}$ & $\begin{array}{c}0.457^{* * *} \\
(5.565)\end{array}$ \\
\hline$S_{i 11}$ & $\begin{array}{c}0.730^{* * *} \\
(8.656)\end{array}$ & $\begin{array}{c}0.218^{* * *} \\
(3.286)\end{array}$ & $\begin{array}{c}0.466^{* * *} \\
(3.116)\end{array}$ & $\begin{array}{l}0.197^{*} \\
(1.910)\end{array}$ & $\begin{array}{c}0.532^{* * *} \\
(5.770)\end{array}$ & $\begin{array}{c}0.450^{* * *} \\
(4.127)\end{array}$ \\
\hline$R^{2}$ & 0.776 & 0.649 & 0.734 & 0.816 & 0.897 & 0.915 \\
\hline $\begin{array}{c}\text { Adjusted } \\
R^{2}\end{array}$ & 0.727 & 0.558 & 0.631 & 0.765 & 0.875 & 0.889 \\
\hline AIC & -0.338 & -1.352 & -1.428 & -0.519 & -1.918 & -1.791 \\
\hline SIC & 0.208 & -0.725 & -0.597 & 0.135 & -1.377 & -1.070 \\
\hline F-statistic & 15.813 & 7.091 & 7.125 & 15.940 & 40.420 & 34.137 \\
\hline D-W & 2.099 & 1.858 & 2.026 & 2.007 & 1.987 & 1.966 \\
\hline$L M_{s c}$ & 1.858 & 1.196 & 3.260 & 0.463 & 0.003 & 4.313 \\
\hline $\mathrm{ARCH}$ & 0.696 & 1.792 & 4.437 & 2.932 & 0.333 & 1.901 \\
\hline $\mathrm{JB}$ & $703.958^{* * *}$ & 1.367 & 1.708 & $6.332^{* *}$ & $28.562^{* * *}$ & 0.795 \\
\hline
\end{tabular}

\subsection{The inbound tourism demand impact}

The direct effect of air pollution is to lower the number of inbound tourists. When potential tourist is thought to be hazardous to their health, air pollution can be expected to play a significant part in decreasing tourist numbers. Hence, the coefficient of dummy DAQI in the model proposed in this research, which has the explanation of a percentage change in the number of inbound tourist arrivals, is anticipated to be significant and negative. According to compare the fitted numbers of inbound tourist arrivals when the dummy DAQI takes different values, the results of percentage change in tourist numbers affected by air pollution which are shown in Table 3, are derived. Although their magnitude approximates the coefficients on the dummy DAQI, it is also smaller and more precise (Song et.al, 2012) [10]. This study assume that long-run relationship between tourism 
demand and macroeconomic determinants would continue and air pollution would no longer be added. Under this assumption, the percentage change in tourist number affected by air pollution is estimated.

Table 3. Percentage change in international tourist arrivals affected by air pollution

\begin{tabular}{|c|c|c|}
\hline & Beijing & Shanghai \\
\hline Canada & $-11.0 \%$ & - \\
\hline Korea & - & $-4.3 \%$ \\
\hline Japan & $-8.8 \%$ & $-10.1 \%$ \\
\hline Thailand & $-5.4 \%$ & - \\
\hline America & $-5.8 \%$ & - \\
\hline UK & $-7.2 \%$ & $-1 \%$ \\
\hline
\end{tabular}

Notes: Calculation is done by comparing the fitted numbers of tourist arrivals (Table 5-6) when the dummy equals 1 and 0.

The results of Table 3 imply that air pollution is present during periods, given that the coefficients are statistically significant. The results illustrate that Beijing air pollution during the study period caused a decrease of inbound tourist number between $5.4 \%$ and $11 \%$ across six major origins. For Beijing, the biggest percentage change in inbound tourist number is Canada, while Thailand is the minimum percentage change of source country. Korean tourist seems to be insensitive to Beijing's air pollution. The air pollution resulted in a loss of Shanghai's tourist arrivals rang $4.4 \%$ to $10.1 \%$ across major source countries. Thailand is the source market most influenced. Moreover, the percentage changes in international tourist arrivals from Asian countries are much larger. This result also suggest that Thai tourists are most sensitive to air pollution in Shanghai, while some market segments (Canada, UK and USA) seemed less sensitive to air pollution of Shanghai. Beijing's inbound tourism demand has a greater impact on air pollution than Shanghai. Since Beijing's air pollution is more serious, the result is consistent with what was expected.

\subsection{The economic impact}

The loss of international tourism receipts is the main concern related to the loss of inbound tourist number. Although the contribution of tourism revenue to China's GDP is negligible, only about $1.2 \%$ in the past 12 years. But the effect of economic has been even larger, because the inbound tourism industry involved all sectors of the economy. Some researchers have pointed that any loss of inbound tourism receipts potentially implies a threefold or fourth fold loss in the whole economy of China (Hai, et.al 2004; Song, et.al, 2012) [10-11].

When we assess the tourism's economic impact, direct effect and secondary effect should be discriminated. This study uses the multiplier effect to indicate the correlation between direct effect and total impact. Thus, in order to size up compute the final economic impact of air pollution, we should determine not only the loss of tourist numbers, but also the potential loss of the whole GDP. Follow Song et.al (2012) [10], the approach is as follow:

Economic impact of Tourism $=$ Tourist Numbers $\times$ Average Spending per visitor $\times$ Multiplier 
Based on the ultimate model specifications achieved in Table 1 and Table 2, this study computes the amount of loss in the number of tourists by computing the difference of fitted of tourist numbers when air pollution dummy DAQI take different numerical values; then, we add up all losses from the six origins. This research applies average international tourism receipts to capture tourist spending. Because the inbound tourism receipts published by tourism administrations do not distinguish across different source markets, so this research use the average international receipts for all source countries. The estimate of lower and upper bound of the multipliers are set at 2 and 4, respectively, which follow WTTC and Song, et.al (2012) [10]. The estimate of multiplies Economic losses of Beijing and Shanghai's inbound tourism (from six major origins) affected by air pollution are shown in Table 4.

Table 4. Economic losses of Beijing and Shanghai's inbound tourism (from six major origins) affected by air pollution

\begin{tabular}{|c|c|c|}
\hline & Beijing & Shanghai \\
\hline Tourist arrivals(1) & -1569.7 & -1167.825 \\
(thousand persons) & & \\
\hline Tourism receipts per capital(2) & 6.539 & 5.226 \\
\hline (thousand yuan) & & \\
\hline Tourism receipts(3)=(1)*(2) (million & -10264.268 & -6103.053 \\
\hline Multiplier(4) & & \\
\hline Lower & 20528.536 & 12206.106 \\
\hline Upper & 41057.072 & 24412.212 \\
\hline Lower(million yuan)
\end{tabular}

Notes: (a) Loss of tourist arrivals, i.e. (1), is calculated by adding up the differences in tourist arrivals across 6 origins when the regulation dummy (DAQI) equals to 1 and 0; (b) Source of tourism receipts per capita, i.e. (1), is the Yearbook of Beijing/Shanghai Tourism Statistics.

The table 4 implies that air pollution of Beijing lowers tourist number of up to 156,970 persons from six major source markets, causing in losses of CNY 10264.27 million in tourism receipts. For Shanghai, the losses of tourist numbers from the same source markets are evaluated to be 1167,825 persons, signifying a loss of CNY 6103.053 million in foreign receipts. The economic impact (Economy GDP) of air pollution rang from CNY 2666.044 to 5332.088 million in Beijing. For Shanghai, the loss from the whole GDP is between CNY 2635.028 and 5270.056 million.

Thus, generally, the two cities of air pollution have already given rise to massive losses to their tourism industry across six major origins, let alone all other foreign source markets to investigate. In additional, if nationwide across all source countries are investigated the amount of loss should be much larger. 


\section{Concluding remarks and suggestion}

The objective of this present paper is to investigate how air pollution potential affects China's inbound tourism demand, in the case of Beijing and Shanghai. The key finding is that air pollution adversely affects China's inbound tourism, resulting in evident loss of tourist number and tourism receipts. Specific for, during the study period, air pollution in Beijing causes a decline in tourist number between $5.4 \%$ and $11 \%$ across six major source markets, suggesting roughly a loss in tourist number of 1569,700 persons, equal to CNY 10264.268 million in tourism receipts. In the case of Shanghai, ratio of tourist number drops ranging from $4.4 \%$ to $10.1 \%$. The loss of tourist number is computed to be 1167,825 persons, implying a loss of CNY 6103.053 million in tourism receipts. Besides, the economic impact (Economy GDP) of air pollution has been also examined, economic losses range from CNY 20528.536 to 41057.072 and 12206.106 to 24412.212 million for Beijing and Shanghai respectively. Noteworthy that this study just investigates the loss of Beijing and Shanghai across six major source countries, the loss should be much greater taking nationwide and all source countries into account. As a result, the effect of air pollution on China's inbound tourism market and economy, as could be for any other country, is too great to be overlooked.

Based on the above conclusions, this paper suggests that the government should pay close attention to the harm of air pollution to inbound tourism and make air pollution control a priority of sustainable tourism development in future. From the perspective of tourism, the diversified tourism market in China, such as the establishment of indoor tourism projects, can be developed, at the same time, the quality of tourism services can be improved to enhance the inbound tourists' satisfaction, which can minimize the effect of air pollution on tourism. Besides, the government should establish an air pollution early warning mechanism, hence realizing real-time adjustment of inbound tourism policy according to the status of air pollution, and the loss of tourist arrivals and reception can be avoided evidently consequently. In terms of air pollution control, logical and technical measures should be taken simultaneously to reduce or even remove air pollution, which means the government should optimize the industrial structure, reduce dependence on the manufacturing sector, enhance the development of non-smoking industries, and take political and financial measures to support the efforts of polluting enterprises in innovative environmental technology. Besides, public awareness of environmental protection should be strengthened in order to promote low-carbon travel (automobile exhaust is one of the causes of air pollution).

Future studies also call on further concentrate upon the effect of air pollution on the attitude of the consumer and country-brand equity. Second, it will be interesting to use the impulse response function in VAR model by daily data of air pollution and tourist arrival to analyze the effect of air pollution on inbound tourist number or reception in different periods. Moreover, analysis of the effect of air pollution on tourists who belong to different market segments (market segments division by age, purpose of visit and so on) can also be taken into account.

Author Contributions: Conceptualization, methodology, writing - review and editing, H. Q. Ma.; investigation, writing-original draft preparation, data curation and supervision, $\mathrm{X}$. Ni.

Funding: This research received no external funding.

Institutional Review Board Statement: Not applicable.

Informed Consent Statement: Informed consent was obtained from all subjects involved in the study.

Data Availability Statement: Not applicable

Conflicts of Interest: The authors declare no conflict of interest. 


\section{References}

1. Algieri, B. An econometric estimation of the demand for tourism: The case of Russia. Tourism Economics. 2006, 12, 5-20.

2. Hamilton J M, Tol RS J. The impact of climate change on tourism in Germany, the UK and Ireland: A simulation study. Regional Environmental Change, 2007, 7 (Suppl. 3), 161- 172.

3. Álvarez-Díaz, M., Otero Giráldez, M.S., González-Gómez, M. Statistical relationships between the North Atlantic Oscillation and international tourism demand in the Balearic Islands. Climate Research. 2010, 43, 207-214.

4. Sajjad, F., Noreen, U., Zaman, K. Climate change and air pollution jointly creating nightmare for tourism industry. Environmental Science and Pollution Research. 2014, 21, 12403-12418.

5. Zhang, A., Zhong, L., Xu, Y. Investigating potential tourists' perception of haze pollution's impacts on tourism experience as to Beijing, China. Advanced Materials Research. 2015, 37, 378-382.

6. Becken, S., Jin X.,Zhang C., Gao J. Urban air pollution in China: destination image and risk perceptions. Journal of Sustainable Tourism. 2016, 25 (Suppl.1), 130-147.

7. Xu, X., Reed, M. Perceived pollution and inbound tourism in China. Tourism Management Perspectives. 2016, 21,109-112.

8. Chen, C.M., Lin, Y.L., Hsu, C.L. Does air pollution drive away tourists? A case study of the Sun Moon Lake National Scenic Area, Taiwan.Transportation Research Part D. 2017, 53, 398-402.

9. Song, H., \& Li, G. Tourism demand modeling and forecasting: a review of recent research. Tourism Management. 2008, 29, 203220.

10. Song, H., Gartner, W.C., Tasci, A.D.A. Visa restrictions and their adverse economic and marketing implications e Evidence from China, Tourism Management. 2012, 33, 397-412.

11. Hai, W., Zhao, Z., Wang, J., \& Hou, Z. The short-term impact of SARS on the Chinese economy. Asian Economic Papers. 2004, 3(Suppl.1), 57-61. 